\title{
A Patient with Takayasu's Arteritis and Rheumatoid Arthritis Who Responded to Tacrolimus Hydrate
}

\author{
Isamu Yokoe, Hitomi Haraoka and Hideaki Harashima
}

\begin{abstract}
We encountered the rare case of a 50-year-old woman who developed rheumatoid arthritis (RA) while suffering from Takayasu's arteritis of arch vessel type. prednisolone (PSL) therapy was continued at a maintenance dose of $7.5 \mathrm{mg}$ due to recurring inflammation. She was affected with RA for 7 years after Takayasu's arteritis. Disease-modifying antirheumatic drugs (DMARDs) were unusable because of side effects. In the summer of 2005, RA activity increased, and treatment with tacrolimus hydrate at $1.5 \mathrm{mg}$ was started; thereafter, the activity of RA and Takayasu's arteritis was relieved, especially MRA findings. We report that therapy with tacrolimus hydrate markedly relieved two disorders, and review the literature.
\end{abstract}

Key words: tacrolimus hydrate, Takayasu's arteritis, rheumatoid arthritis

(DOI: 10.2169/internalmedicine.46.0211)

\section{Introduction}

Takayasu's arteritis is a chronic inflammatory disorder involving the aorta and its branches. Vascular stenosis and occlusion cause various clinical symptoms. This arteritis is frequent among young women. Optimal-dose steroid therapy inhibits the inflammatory response, showing a good prognosis. However, in some patients in whom control is poor, it is difficult to keep a balance between the maintenance dose of steroid and side effects such as Cushing's syndrome. In particular, the selection of treatment is important in patients with this arteritis and other types of collagen disease. In this study, we report a patient in whom rheumatoid arthritis (RA) developed during the course of Takayasu's arteritis, and therapy with tacrolimus hydrate markedly relieved the two disorders, and review the literature.

\section{Case Report}

The patient was a 50-year-old woman. In 1996, general malaise, fever, and a decrease in the right radial pulse were noted. Computed tomography (CT) angiography suggested aortic arch vessel-type Takayasu's arteritis. Therapy with PSL at $30 \mathrm{mg}$ was started (Fig. 1).

As shown in the figure, the patient started to undergo treatment in 1996, but inflammation repeatedly occurred with decreases in the dose of PSL. In 1999, since CRP increased in addition to fever and vertigo, the dose of PSL was increased to $20 \mathrm{mg}$. Mild inflammation repeatedly occurred, and the dose of PSL could not be decreased to less than $7.5 \mathrm{mg}$. The CRP level was about $1.0 \mathrm{mg} / \mathrm{dl}$, and never became negative. However, symptoms, such as arthritis, were not detected. In 2003, morning stiffness and symmetric arthritis involving the right wrist joint were observed. The patient was positive for rheumatoid factor (RF). Radiography showed bone atrophy around the wrist, suggesting RA. Simultaneously, magnetic resonance angiography (MRA) did not reveal any vascular stenotic lesion. Methotrexate (MTX) at $6 \mathrm{mg}$ was additionally administered, but was discontinued due to severe gastrointestinal disorders. Other diseasemodifying antirheumatic drugs (DMARDs) such as salazosulfapyridine and bucillamine were administered. However, side effects such as exanthema and proteinuria occurred, and these agents were discontinued. In the summer of 2005, RA activity increased, and treatment with tacrolimus hydrate at $1.5 \mathrm{mg}$ was started in December. Polyarthritis, inflammation based on hematological data, and right radial pulse reduction was relieved. In July 2006, there were no changes in occlusion at the bifurcation of the right subclavian artery on MRA in comparison to the MRA findings in 2005. However, the distal subclavian, internal carotid, and vertebral ar- 
Table 1. Comparison of the Hematological Data before and after Treatment with Tacrolimus Hydrate

Examination findings <Comparison between before and after administering>

\begin{tabular}{|c|c|c|c|c|c|c|c|c|c|}
\hline \multicolumn{3}{|c|}{ December, 2005} & \multicolumn{2}{|c|}{ February, 2006} & \multicolumn{3}{|c|}{ December, 2005} & \multicolumn{2}{|c|}{ February, 2006} \\
\hline WBC : & 10800 & $\rightarrow$ & 8000 & $/ \mu \ell$ & TP : & 7.8 & $\rightarrow$ & 7.5 & $g / d \ell$ \\
\hline Nuet : & 87.6 & $\rightarrow$ & 78.1 & $\%$ & GOT : & 16 & $\rightarrow$ & 9 & IU/L \\
\hline Eos : & 0.2 & $\rightarrow$ & 0.4 & $\%$ & GPT : & 11 & $\rightarrow$ & 14 & IU/L \\
\hline Baso : & 0.3 & $\rightarrow$ & 0.2 & $\%$ & Т-СНО : & 187 & $\rightarrow$ & 176 & $\mathrm{mg} / \mathrm{d} \ell$ \\
\hline Mono : & 0.7 & $\rightarrow$ & 2.5 & $\%$ & TG : & 145 & $\rightarrow$ & 126 & $\mathrm{mg} / \mathrm{d} \ell$ \\
\hline Lympho : & 11.2 & $\rightarrow$ & 18.8 & $\%$ & HDL-CHO & 45 & $\rightarrow$ & 52 & $\mathrm{mg} / \mathrm{d} \ell$ \\
\hline RBC : & 409 & $\rightarrow$ & 391 & $10^{4} / \mu \ell$ & BUN : & 22.1 & $\rightarrow$ & 20.1 & $\mathrm{mg} / \mathrm{d} \ell$ \\
\hline h. & 11.6 & $\rightarrow$ & 11.2 & $\mathrm{~g} / \mathrm{d} \ell$ & $\mathrm{Cr}:$ & 0.71 & $\rightarrow$ & 0.67 & $\mathrm{mg} / \mathrm{d} \ell$ \\
\hline & 35.0 & $\rightarrow$ & 33.9 & $\%$ & CRP : & 1.84 & $\rightarrow$ & 0.37 & $\mathrm{mg} / \mathrm{d} \ell$ \\
\hline 'It & 36.3 & $\rightarrow$ & 28.7 & $10^{4} / \mu \ell$ & BS : & 104 & $\rightarrow$ & 111 & $\mathrm{mg} / \mathrm{d} \ell$ \\
\hline ICV: & 86 & $\rightarrow$ & 87 & fl & HbA1c: & 6.1 & $\rightarrow$ & 5.7 & $\%$ \\
\hline CH : & 28.4 & $\rightarrow$ & 28.6 & Pg & RF : & 12.9 & $\rightarrow$ & 7.7 & $\mathrm{IU} / \mathrm{ml}$ \\
\hline МСНС : & 33.2 & $\rightarrow$ & 32.9 & $\%$ & ESR : & 61 & $\rightarrow$ & 30 & $\mathrm{mg} / \mathrm{d} \ell$ \\
\hline & & & & & MMP-3 : & 153 & $\rightarrow$ & 119 & $\mathrm{ng} / \mathrm{m}$ \\
\hline
\end{tabular}

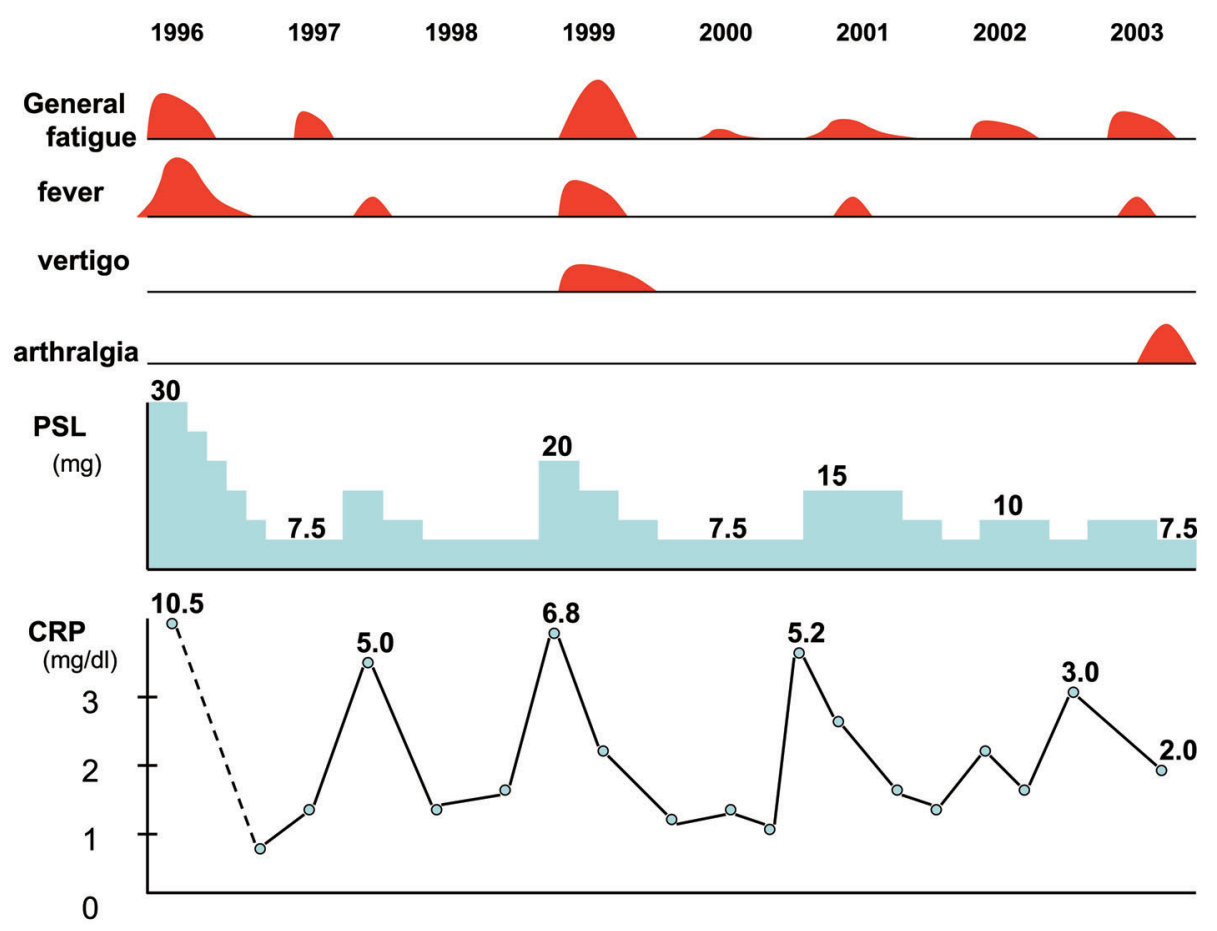

Figure 1. Treatment before the occurrence of RA.

teries were visualized more clearly. On the original images, the degree of hypertrophy of the wall could not be evaluated, but improvement of the luminal diameter was clearly observed (Figs. 2a, 2b, 2c, 2d).

Currently, PSL at $5 \mathrm{mg}$ is administered, and RA activity is controlled with tacrolimus hydrate at $1.5 \mathrm{mg}$. We compared the results before and after the administration of tacrolimus hydrate. Clinically, the symptoms including arthritis were markedly relieved. Before administration, it was impossible to measure blood pressure via the right forearm using a blood pressure meter. On palpation, the blood pressure value was $90 \mathrm{mmHg}$. After administration, it improved to $120 \mathrm{mmHg}$, which was measured using a blood pressure meter. Hematology showed decreases in the white blood cell count (WBC), erythrocyte sedimentation rate (ESR), and C reactive protein (CRP), RF, and MMP-3 levels (Table 1).

Concerning RA, periarticular osteoporosis of the right wrist was suggested at diagnosis. With rapid articular destruction, bone corrosion and narrowing of the articular space were observed before the administration of tacrolimus hydrate (Fig. 3). The bone findings were not improved even after the administration of tacrolimus hydrate. However, arthralgia and arthritis markedly subsided. The DAS28 (ESR) values before and after administration were 5.22 and 2.78 , respectively. This condition is being maintained.

On MRA, the left external carotid, subclavian, and inter- 



Cross-section (2005)

Cross-section (2006)

Figure 2. The left external carotid, subclavian, and internal thoracic arteries (arrows) were visualized more clearly (Before (Fig. 2a, 2c) and after (Fig. 2b, 2d) administration of tacrolimus).

nal thoracic arteries were visualized more clearly, as described above. CT angiography after 1 year of therapy with tacrolimus hydrate revealed complete occlusion at the origin of the right subclavian artery branching from the right brachiocephalic trunk. The artery was visualized as a retrograde route from the right vertebral artery via the collateral pathway. However, there were no other stenotic lesions involving the abdominal aorta (Fig. 4).

\section{Discussion}

Takayasu's arteritis indicates nonspecific large vasculitis, which induces occlusive or dilative lesions in the aorta, its main vessels, and pulmonary/coronary arteries. The pathogenesis remains to be clarified. However, cold-like symptoms are frequently observed as precursory symptoms. In the presence of viral infection, vasculitis may deteriorate via an autoimmune mechanism $(1,2)$.

Aortic magnetic resonance imaging (MRI) may be useful for early diagnosis. Takayasu's arteritis-related inflammation may initially involve the outer membrane, and then gradually the intima side. These changes are difficult to evaluate by plain X-ray or CT. Contrast-enhanced MRI facilitates the early detection of intramural edematous changes. Inflammatory edema becomes less marked in the late stage, resulting in vascular stenosis/occlusion via fibrosis. Steroid therapy in this stage does not reduce vascular stenosis; steroid therapy for inflammatory edema may inhibit the deterioration of vas- cular stenosis. In the present case, complete fibrosis/occlusion was possibly present at the origin of the right subclavian artery, whereas there were possibly edematous changes without complete fibrosis in the left external carotid, subclavian, and internal thoracic arteries, in which MRA showed improvement. This also suggests the importance of diagnosing this disorder, which is frequent among young women, in the early stage.

CRP is considered to be a parameter of activity. Steroid therapy at an optimal dose markedly inhibits the inflammatory response. However, in some patients with refractory Takayasu's arteritis or another type of collagen disease, massive steroid therapy or combination therapy with immunosuppressive agents is required to inhibit the inflammatory response, as demonstrated in the present case, often making treatment difficult.

In the present case, RA developed during the course. Several studies have reported Takayasu's arteritis complicated by systemic lupus erythematosus (SLE) or systemic sclerosis. However, the concurrent development of RA is rare (3, 4). According to Korkmaz et al, the mean age at the diagnosis of Takayasu's arteritis was $55.5 \pm 15$ years (range: $20-82$ ) in 20 patients with Takayasu's arteritis and RA. It was more advanced than the typical age at the diagnosis of Takayasu's arteritis (5). This was possibly because initial symptoms of Takayasu's arteritis, such as slight fever, malaise, and arthralgia, which are also frequent in the presence of RA, were masked as RA-related symptoms. 


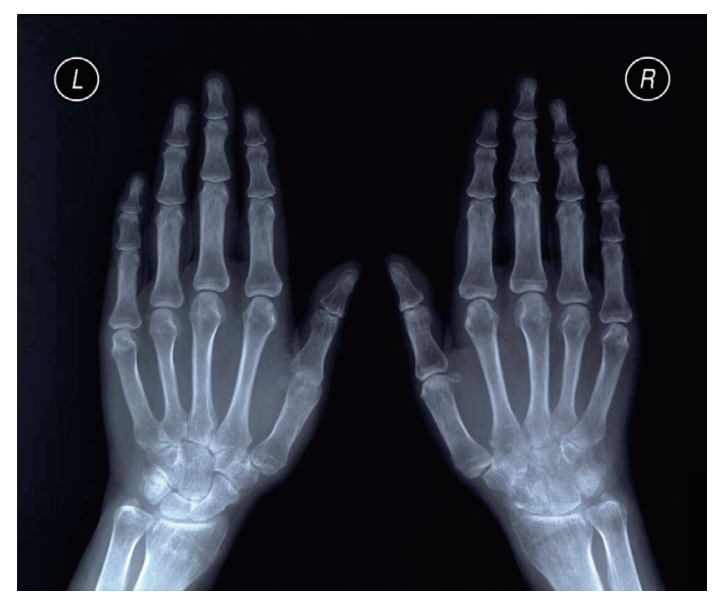

Figure 3. In the right carpal bone, space narrowing, bone corrosion, and fusion were observed (Before treatment with tacrolimus hydrate (December 2005).

In their review, the mean interval from the diagnosis of Takayasu's arteritis until that of RA was 7.5 years. RArelated vascular lesions mainly consist of middle/minor blood vessels. However, aortitis and aortic valve disorders are sometimes observed (6), as demonstrated for other types of collagen disease. According to Gravallese et al, autopsy revealed aortitis or valvular inflammation in $15 \%$ of RA patients without signs of aortic insufficiency (7). Furthermore, the incidence of arthralgia is high during the course of Takayasu's arteritis. Sato et al reported that arthralgia or arthritis occurred in $26 \%$ of their patients during the course of Takayasu's arteritis (8). Hall et al indicated that arthralgia and synovitis were noted in $56 \%$ and $22 \%$ of 32 patients with Takayasu's arteritis, respectively (9).

Thus, the association between Takayasu's arteritis and RA remains to be clarified. However, the two disorders represent hereditary predisposition-associated systemic inflammatory changes. When ischemia-related symptoms appear during the course of RA, or when arthralgia or arthritis occurs during the course of Takayasu's arteritis, close follow-up is needed.

With regard to the improvement of the 2 diseases by tacrolimus hydrate in the present patient, the following mechanism is considered: The Th1 subset, which is mainly related to cell-mediated immune responses, is considered to play a central role in RA and several other rheumatic diseases (10). The generation of T-cell subsets is mostly controlled by certain cytokines. Interleukin (IL)-12, IL-18, and IFN- $\gamma$ enhance differentiation to Th1, while IL-4 and IL-10 enhance differentiation to Th2, and suppress Th1 cells. Therefore, the induction of changes in the Th1/Th2 balance is considered useful for the treatment of RA.

The etiology of Takayasu's arteritis is unclear. However, the pathology of the arterial wall confirmed infiltration of CD8 $\mathrm{T}$ cells. These cytotoxic $\mathrm{T}$ lymphocytes and natural killer cells may contribute to smooth muscle cell disorders. In analogy with rheumatoid arthritis, $\mathrm{T}$ cells infiltrating the vessel walls might release strong pro-inflammatory cyto-

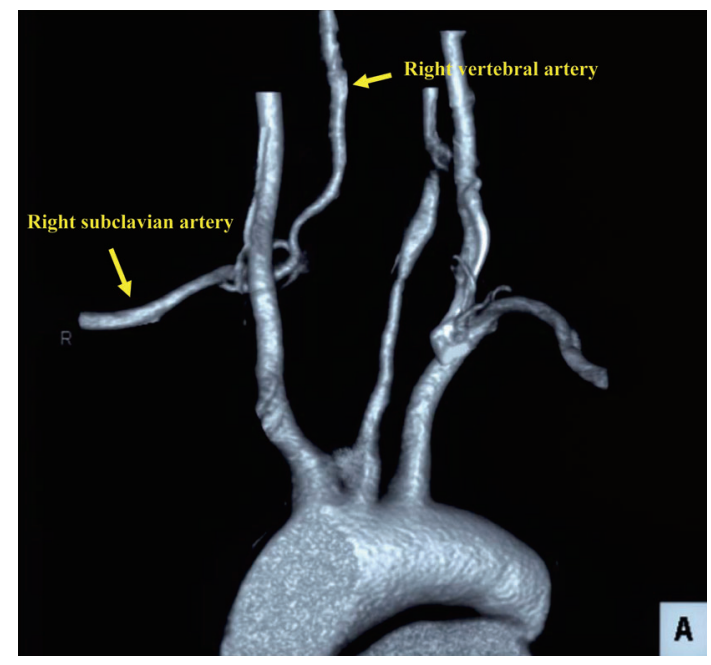

Figure 4. CT angiography. The right subclavian artery was occluded at the bifurcation of the brachiocephalic trunk, showing retrograde feeding from the right vertebral artery (arrows). There was no stenosis in other blood vessels including the vertebral artery or brachiocephalic trunk.

kines, such as IL-6 and tumor necrosis factor (TNF)- $\alpha$ (11).

Takayasu's arteritis is an idiopathic systemic granulomatous disease, and granulomatous inflammation is based in part on TNF. Macrophages, T cells, natural killer cells, and vascular endothelial cells produce TNF. TNF induces macrophage production of IL-12 and IL-18, which are potent cytokines that enhance CD4 $\mathrm{T}$ lymphocyte differentiation to Th1 cells and activate natural killer cells. Subsequently Th1 leads to the recruitment and activation of macrophages. Vessel damage from injury by activated $\mathrm{T}$ cells, macrophages, and natural killer cells is an important pathogenic mechanism of Takayasu's arteritis (12).

With respect to the pharmacological actions of tacrolimus hydrate, it is speculated that this agent suppresses TNF- $\alpha$, interleukin (IL)-2, IL-6, and interferon (IFN)- $\gamma$ expression by inhibiting the activation of nuclear factor of activated $\mathrm{T}$ cell (NFAT), which is important for T-cell activation. These findings suggest that tacrolimus hydrate suppresses the activity of Takayasu's arteritis and RA by inhibiting T-cell activation.

TNF is involved in RA activity, and there have been a number of studies showing the usefulness of TNF- $\alpha$ inhibitors for the treatment of rheumatic diseases. Recently, TNF$\alpha$ inhibitors have been applied in the treatment of Takayasu's arteritis (11-13).

Hoffman et al reported the efficacy of anti-TNF therapy in an open label study in 15 patients with Takayasu's arteritis. Ten of the 15 patients achieved complete remission without glucocorticoid therapy, and four patients achieved partial remission (12).

As described above, molecular-targeted therapy is effective in Takayasu's arteritis and RA. The patient of this study was considered interesting with regard to evaluating the background and etiology of complications of Takayasu's ar- 
teritis and RA. To achieve remission of Takayasu's arteritis and RA, it is sometimes necessary to administer a large amount of steroids, which is often accompanied by unacceptable side-effects, such as Cushing's syndrome. The administration of tacrolimus hydrate and anti-TNF therapy, which are useful as adjuvant treatment methods, can help to reduce or discontinue steroid administration, leading to resolution of long-term side effects.

\section{Conclusion}

In the present patient, it was impossible to administer DMARDs such as MTX due to side effects. Considering the activity of Takayasu's arteritis, tacrolimus hydrate was selected. This agent relieved Takayasu's arteritis and RA, suggesting its usefulness in the presence of the two disorders. In the future, the effects of tacrolimus hydrate on the concurrent development of Takayasu's arteritis and RA or other types of collagen disease should be investigated in a larger number of patients.

\section{References}

1. Desiron Q, Zeaiter R. Takayasu's arteritis. Acta Chir Belg 100: 16, 2000.

2. Numano F, Okawara M, Inomata H, Kobayashi Y. Takayasu's arteritis. Lancet 356: 1023-1025, 2000.

3. Igarashi $T$, Nagaoka $S$, Matsunaga $K$, et al. Aortitis syndrome (Takayasu's arteritis) associated with systemic lupus erythematosus. J Rheumatol 16: 1579-1583, 1989.

4. Passiu G, Vacca A, Sanna G, et al. Takayasu's arteritis overlapping with systemic sclerosis. Clin Exp Rheumatol 17: 363-365, 1999.

5. Korkmaz C, Zubaroglu I, Kaya T, Akay OM. Takayasu's arteritis associated with rheumatoid arthritis: a case report and review of the literature. Rheumatology 40: 1420-1422, 2001.

6. Townend JN, Emery P, Davies MK, Littler WA. Acute aortitis and aortic incompetence due to systemic rheumatological disorders. Int J Cardiol 33: 253-258, 1991.

7. Gravallese EM, Corson JM, Coblyn JS, Pinkus GS, Weinblatt ME. Rheumatoid aortitis: a rarely recognized but clinically significant entity. Medicine 68: 95-106, 1989.

8. Sato EI, Hatta FS, Levy-Neto M, Fernandes S. Demographic, clinical, and angiographic data of patients with Takayasu arteritis in Brazil. Int J Cardiol 66: 67-70, 1998

9. Hall S, Barr W, Lie JT, Stanson AW, Kazmier FJ, Hunder GG. Takayasu arteritis. A study of 32 North American patients. Medicine 64: 89-99, 1985.

10. Miossec P, van den Berg W. Th1/Th2 cytokine balance in arthritis. Arthritis Rheum 40: 2105-2115, 1997.

11. Della Rossa A, Tavoni A, Merlini G, et al. Two Takayasu arteritis patients successfully treated with infliximab: a potential diseasemodifying agent? Rheumatology 44: 1074-1075, 2005.

12. Hoffman GS, Merkel PA, Brasington RD, Lenschow DJ, Liang P. Anti-tumor necrosis factor therapy in patients with difficult to treat Takayasu arteritis. Arthritis Rheum 50: 2296-2304, 2004.

13. Jolly M, Curran JJ. Infliximab-responsive uveitis and vasculitis in a patient with Takayasu arteritis. J Clin Rheumatol 11: 213-215, 2005 .

(C) 2007 The Japanese Society of Internal Medicine http://www.naika.or.jp/imindex.html 